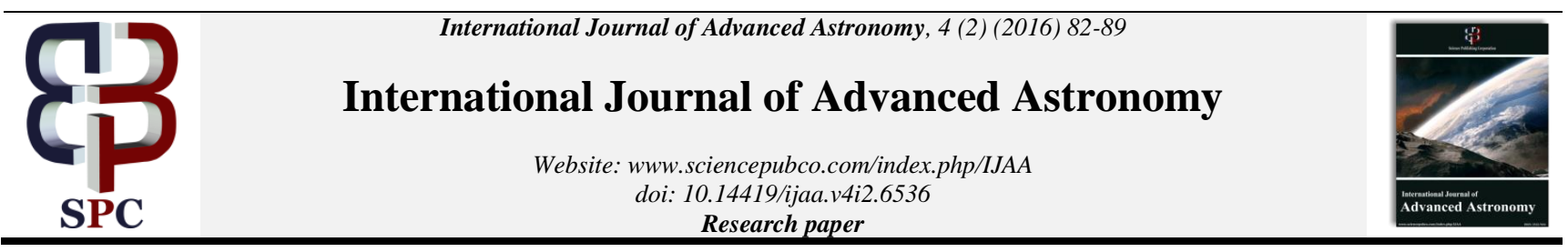

\title{
Linear stability and resonance of triangular equilibrium points in elliptic restricted three body problem with radiating primary and triaxial secondary
}

\author{
T. Usha *, A. Narayan \\ ${ }^{1}$ Department of Applied Mathematics, Bhilai Institute of Technology, Durg, India \\ *Corresponding authorE-mail: usha156@gmail.com, ashutoshmaths.narayan@gmail.com
}

\begin{abstract}
The present paper studies the linear stability of the triangular equilibrium points of the system. The system comprises of a radiating primary and a triaxial secondary in elliptic restricted three body problem. The existence of third order resonances has been shown and the linear stability has been analyzed for these resonance cases. For the resonance case, $3 \lambda_{2}=1$ and $2 \lambda_{1}+\lambda_{2}=0$, the conditions of the linear stability are satisfied and the system is stable. But, for the resonance cases $2 \lambda_{1}+\lambda_{2}=1$ and $\lambda_{1}-2 \lambda_{2}=2$ the system is unstable.
\end{abstract}

Keywords: Celestial Mechanics; Elliptical Restricted Three Body Problem; Stability; Oblateness; Rigid Body; Resonance.

\section{Introduction}

The elliptic restricted three body problem has been widely studied by many researchers which is a generalization of classical model. The system consists of two finite bodies (known as primaries) moving about their common center of mass, having no influence on each other. The third body is influenced by both of the primaries. The primaries in general describe elliptic path. The orbit of Jupiter around Sun is a fixed ellipse and the Trojan asteroids are influenced by the gravitational attraction of the Sun and Jupiter is an example of the above system. The study of restricted/elliptic restricted three body problem has been a subject of investigation over the years. The stability of such systems (ER3BP) moving in an elliptic orbits was subject of investigation and was investigated by many authors, Arnold [1] ; Danby [11]; Bennet [4]; Szebehely [25]; Broucke [6]; Katsiaris [19] ; Beauge[5]; Baoyin[3]; Ammar[2]; Biggs [7]; Biggs [8] and many others.

The resonance/non-resonance cases of libration points for restricted/elliptic restricted three body problem was studied and analyzed by many authors Kamel [18]; Choudhry [10]; Ferraz [12]; Henrard [15]; Kumar [20]; Henrard [17], Hadjidemetriou [13]; Hadjidemetriou [14]; Subba Rao [24]; Thakur [26]; Beauge [5]; Chandra [9]; Narayan [22] and many others.

In the present paper an attempt have been made to study and analyze the linear stability of the system. It has been shown that resonances of the third order exist under the range of linear stability. This paper is in continuation of the paper ( Usha [27] and Narayan [23]). The study of linear stability in presence of resonance makes the work different from the other works. We have followed the method proposed by Kumar [20].

The present paper is organized as follows: Section 1, which is introduction; Section 2 provides the equations of motion; Section 3 gives the location of the Triangular points; Section 4 focuses on the First order stability and Normalization of Hamiltonian and
Section 5 gives the Resonance Cases. The discussions and conclusions are drawn in Section 6.

\section{Equations of motion}

The equations of motion of the infinitesimal mass in the elliptic restricted three body problem under radiating and triaxial primaries in the barycentric, pulsating and rotating, non-dimensional coordinates are given by the differential equations derived in Usha et. al. [27] and given in the following equation (1). The notations in principle follow the book of Szebehely [25], with some minor modifications in the notation being done for adapting to the present problem, presented as:

$$
\begin{aligned}
& x^{\prime \prime}-2 y^{\prime}=\frac{1}{1+e \cos v} \frac{\partial \Omega}{\partial x} \\
& y^{\prime \prime}+2 x^{\prime}=\frac{1}{1+e \cos v} \frac{\partial \Omega}{\partial y}
\end{aligned}
$$

where ' denotes differentiation with respect to $v$, and

$$
\Omega=\frac{x^{2}+y^{2}}{2}+\frac{1}{n^{2}}\left[\begin{array}{l}
\frac{(1-\mu) q}{r_{1}}+\frac{\mu}{r_{2}} \\
+\frac{\mu\left(2 \sigma_{1}-\sigma_{2}\right)}{2 r_{2}^{3}}-\frac{3 \mu\left(\sigma_{1}-\sigma_{2}\right)}{2 r_{2}^{5}} y^{2}
\end{array}\right]
$$

where $\mathrm{n}^{2}=1+\frac{3}{2}\left(2 \sigma_{1}-\sigma_{2}\right) \quad$ and $\sigma_{1}=\frac{\mathrm{a}^{2}-\mathrm{c}^{2}}{5 \mathrm{R}^{2}} ; \quad \sigma 2=\frac{\mathrm{b}^{2}-\mathrm{c}^{2}}{5 \mathrm{R}^{2}}$. and 


$$
\mathrm{r}_{1}^{2}=(\mathrm{x}+\mu)^{2}+\mathrm{y}^{2}, \mathrm{r}_{2}^{2}=(\mathrm{x}-1+\mu)^{2}+\mathrm{y}^{2}
$$

Here $m_{1}$ and $m_{2}$ are the masses of the bigger and smaller primaries positioned at $\left(x_{i}, 0\right), \mathrm{i}=1,2 ; q=1-\delta$, the radiation pressure; $\sigma_{1}$ and $\sigma_{2}$ are triaxiality parameters, $\sigma_{i}(\mathrm{i}=1,2)$ (McCuskey [21]); and a, b, c are semi axes and $\mathrm{R}$ is the distance between the primaries ; $r_{i}(\mathrm{i}=1,2)$ are the distances of the infinitesimal mass from the bigger and smaller primaries respectively; while $e$ is the eccentricity of the either primary around the other and $v$ is the true anomaly.

\section{Location of triangular equilibrium points}

The equilibrium points of the system are given by the equations:

$$
\begin{aligned}
& \frac{\partial \Omega}{\partial x}=0 \\
& \frac{\partial \Omega}{\partial y}=0
\end{aligned}
$$

where $\Omega$ is given by equation (2).

$$
\frac{\partial \Omega}{\partial x}=x-\frac{1}{n^{2}}\left\{\begin{array}{l}
\frac{(1-\mu)(x+\mu) q}{r_{1}^{3}} \\
+\frac{\mu(x-1+\mu)}{r_{2}^{3}} \\
+\frac{3 \mu(x-1+\mu)\left(2 \sigma_{1}-\sigma_{2}\right)}{2 r_{2}^{5}} \\
-\frac{15 \mu(x-1+\mu)\left(\sigma_{1}-\sigma_{2}\right) y^{2}}{2 r_{2}^{7}}
\end{array}\right\}=0
$$

and

$$
\frac{\partial \Omega}{\partial y}=y\left[1-\frac{1}{n^{2}}\left\{\begin{array}{c}
\frac{(1-\mu) q}{r_{1}^{3}+\frac{\mu}{r_{2}^{3}}} \\
+\frac{3 \mu\left(4 \sigma_{1}-3 \sigma_{2}\right)}{2 r_{2}^{5}} \\
-\frac{15 \mu\left(\sigma_{1}-\sigma_{2}\right) y^{2}}{2 r_{2}^{7}}
\end{array}\right\}\right]=0
$$

Solving the above equations, the coordinates of the triangular libration points $L_{4,5}$ are represented as:

$$
x=\left[\begin{array}{l}
\mu-\frac{1}{2}-\frac{(1-q)}{3} \\
+\left\{\frac{3}{8}-(1-q)+\frac{\mu}{2(1-\mu)}\right\} \sigma_{1} \\
+\left\{\frac{-7}{8}+\frac{(1-q)}{2}-\frac{\mu}{2(1-\mu)}\right\} \sigma_{2}
\end{array}\right]
$$

$$
y= \pm \frac{\sqrt{3}}{2}\left[1+\frac{2}{3}\left\{\begin{array}{l}
\frac{(q-1)}{3} \\
+\left(\frac{-19}{8}-(1-q)+\frac{\mu}{2(1-\mu)}\right) \sigma_{1} \\
+\left(\frac{15}{8}+\frac{(1-q)}{2}-\frac{\mu}{2(1-\mu)}\right) \sigma^{2}
\end{array}\right\}\right]
$$

\section{First order stability and normalization of Hamiltonian}

The Lagrangian equation of motion of the problem is written as:

$$
\begin{aligned}
& L=\frac{1}{2}\left(\dot{x}^{2}+\dot{y}^{2}\right)+(x \dot{y}-y \dot{x}) \\
& +\frac{1}{1+e \cos v}\left\{\begin{array}{c}
\frac{x^{2}+y^{2}}{2} \\
+\frac{1}{n^{2}}\left[\begin{array}{c}
\frac{(1-\mu) q}{r_{1}}+\frac{\mu}{r_{2}} \\
+\frac{\mu\left(2 \sigma_{1}-\sigma_{2}\right)}{2 r_{2}^{3}} \\
-\frac{3 \mu\left(\sigma_{1}-\sigma_{2}\right)}{2 r_{2}^{5}} y^{2}
\end{array}\right]
\end{array}\right\}
\end{aligned}
$$

Now, to get the Hamiltonian function of the problem, using the formula:

$$
H=-L+\frac{\partial L}{\partial \dot{x}} \dot{x}+\frac{\partial L}{\partial \dot{y}} \dot{y}
$$

Hence, the perturbed Hamiltonian is given by:

$$
\begin{aligned}
& H=\frac{1}{2}\left(P_{x}^{2}+P_{y}^{2}\right)+\left(P_{x} y-P_{y} x\right) \\
& +\frac{e \cos v}{1+e \cos v}\left(x^{2}+y^{2}\right)
\end{aligned}
$$

$$
-\frac{1}{1+e \cos v}\left\{\frac{1}{n^{2}}\left[\begin{array}{c}
\frac{(1-\mu) q}{r_{1}}+\frac{\mu}{r_{2}} \\
+\frac{\mu\left(2 \sigma_{1}-\sigma_{2}\right)}{2 r_{2}^{3}} \\
-\frac{3 \mu\left(\sigma_{1}-\sigma_{2}\right)}{2 r_{2}^{5}} y^{2}
\end{array}\right]\right\}
$$

where $P_{x}$ and $P_{y}$ are the generalized components of momentum. The nature of motion near the two points will be the same as the two triangular equilibrium solutions are symmetrical to each other. Hence, in further calculations the motion near the equilibrium point $L_{4}$ will be considered.

So, shifting the origin to $L_{4}$ by the change of variables given by:

$$
\begin{aligned}
& x=\xi+q_{1} ; \\
& y=\eta+q_{2} ; \\
& P_{x}=P_{\xi}+P_{1} ; \\
& P_{y}=P_{\eta}+P_{2} .
\end{aligned}
$$


So, the point $L_{4}$ is given by:

$$
\begin{aligned}
& \xi=\frac{1}{2}-\mu-\frac{\delta}{3}+\left\{\frac{3}{8}-\delta+\frac{\mu}{2(1-\mu)}\right\} \sigma_{1} \\
& +\left\{\frac{-7}{8}+\frac{\delta}{2}-\frac{\mu}{2(1-\mu)}\right\} \sigma_{2} ; \\
& \eta=\frac{\sqrt{3}}{2}\left[1+\frac{2}{3}\left\{\begin{array}{l}
\frac{-\delta}{3}+\left(\frac{-19}{8}-\delta+\frac{\mu}{2(1-\mu)}\right) \sigma_{1} \\
+\left(\frac{15}{8}+\frac{\delta}{2}-\frac{\mu}{2(1-\mu)}\right) \sigma 2
\end{array}\right\} ;\right. \\
& P_{\xi}=-\eta ; \\
& P_{\eta}=\xi .
\end{aligned}
$$

The solution of the equation (12) in the new variables are given by the equilibrium position:

$q_{1}=q_{2}=P_{1}=P_{2}=0$

Now, expanding the Hamiltonian function (10) in the powers of $P_{i}$ and $q_{i}, \mathrm{i}=1,2$, we obtain:

$H=\sum_{k=0}^{\infty} H_{k}=H_{0}+H_{1}+H_{2}+H_{3}+H_{4}+\ldots \ldots$

where $H_{0}=$ constant and $H_{1}=0$.

Taking only $\mathrm{H}_{2}$, as we are analyzing the linear stability, we have:

$$
\begin{aligned}
& H_{2}=\frac{1}{2}\left(p_{1}^{2}+p_{2}^{2}\right)+\left(p_{1} q_{2}-q_{1} p_{2}\right) \\
& +\frac{e \cos v}{1+e \cos v}\left(q_{1}^{2}+q_{2}^{2}\right) \\
& +\frac{1}{2(1+e \cos v)}\left[A q_{1}^{2}-B q_{1} q_{2}-C q_{2}^{2}\right]
\end{aligned}
$$

The above equation can be written as:

$$
H_{2}=H_{2}{ }^{(0)}+H_{2}{ }^{(1)}
$$

where,

$$
\begin{aligned}
& H_{2}{ }^{(0)}=\frac{1}{2}\left(p_{1}^{2}+p_{2}^{2}\right)+\left(p_{1} q_{2}-q_{1} p_{2}\right) \\
& +\frac{1}{2}\left\{A q_{1}^{2}-B q_{1} q_{2}-C q_{2}^{2}\right\} \\
& H_{2}{ }^{(1)}=\frac{e \cos v}{1+e \cos v}\left\{\begin{array}{l}
(1-A) q_{1}^{2} \\
+B q_{1} q_{2}+(1+C) q_{2}^{2}
\end{array}\right\}
\end{aligned}
$$

and,

$$
\begin{aligned}
& A=\frac{1}{8}+\frac{\delta}{4}+\left(\frac{-21}{16}+\frac{3}{64} \mu\right) \sigma_{1}+\left(\frac{27}{16}-\frac{99}{64} \mu\right) \sigma_{2} \\
& B=\frac{\sqrt{3}}{4}\left\{\frac{3}{4}-\frac{3 \mu}{32}-\frac{2 \delta}{3}+\left(\frac{7}{2}-\frac{21}{8} \mu\right) \sigma_{1}+\left(\frac{-9}{2}+\frac{181}{8} \mu\right) \sigma_{2}\right\} \\
& C=\frac{5}{8}+\frac{\delta}{4}-\frac{3 \mu \delta}{4}+\left(\frac{-21}{16}+\frac{183}{64} \mu\right) \sigma_{1}+\left(\frac{27}{16}-\frac{183}{64} \mu\right) \sigma_{2}--
\end{aligned}
$$

For normalizing using the canonical transformation:

$$
\left[q_{1}, q_{2}, p_{1}, p_{2}\right]=\left[q_{1}^{\prime}, q_{2}^{\prime}, p_{1}{ }^{\prime}, p_{2}{ }^{\prime}\right] N
$$

where,

$N=\left(\begin{array}{cccc}a_{1} & a_{1} c_{1} & -a_{1} c_{1} & a_{1}\left(1-\omega_{1} b_{1}\right) \\ a_{2} & a_{2} c_{2} & -a_{2} c_{2} & a_{2}\left(1-\omega_{2} b_{2}\right) \\ 0 & a_{1} b_{1} & a_{1}\left(1-b_{1}\right) & a_{1} c_{1} \\ 0 & -a_{2} b_{2} & -a_{2}\left(1-b_{2}\right) & -a_{2} c_{2}\end{array}\right)$

and,

$$
a_{i}=\frac{1}{2} \sqrt{\frac{2 l_{i}}{\left|\omega_{i}^{2}-\frac{1}{2}\right|}} ; b_{i}=\frac{2}{l_{i}} ; \quad c_{i}=\frac{-B}{l_{i}} ;
$$

where, $\mathrm{i}=1,2$ and $\mathrm{A}, \mathrm{B}, \mathrm{C}$ are given by the equation (17). Here, $\omega_{1}^{2}=-\left\{\lambda_{1,2}^{(0)}\right\}^{2}$ and $\omega_{2}^{2}=-\left\{\lambda_{3,4}^{(0)}\right\}^{2}$, are the frequencies and given by the relation:

$$
\begin{aligned}
& \omega^{4}-\left\{1-\frac{45}{8} \mu \sigma_{1}+\frac{21}{8} \mu \sigma_{2}\right\} \omega^{2} \\
& +\frac{27}{4} \mu(1-\mu)\left\{\begin{array}{l}
1+\frac{2}{9} \\
+\left(\frac{71}{8} \sigma_{1}-\frac{181}{24} \sigma_{2}\right)
\end{array}\right\}=0
\end{aligned}
$$

On solving the above equation, we have:

$$
\left.\omega_{1,2}^{2}=\frac{1}{2}\left[\begin{array}{l}
\left\{1-\frac{45}{8} \mu \sigma_{1}+\frac{21}{8} \mu \sigma_{2}\right\} \\
\pm\left\{\left(1-\frac{45}{4} \mu \sigma_{1}+\frac{21}{4} \mu \sigma_{2}\right)\right. \\
-27 \mu(1-\mu)\left(1+\frac{2}{9}+\frac{71}{8} \sigma_{1}-\frac{181}{24} \sigma_{2}\right)
\end{array}\right\}\right]
$$

If $\omega_{1,2}^{2}$ are purely imaginary, we have:

$$
\left\{\begin{array}{l}
\left(1-\frac{45}{4} \mu \sigma_{1}+\frac{21}{4} \mu \sigma_{2}\right) \\
-27 \mu(1-\mu)\left(1+\frac{2}{9}+\frac{71}{8} \sigma_{1}-\frac{181}{24} \sigma_{2}\right)
\end{array}\right\} \geq 0
$$

The equality relation corresponds to resonance cases with equal frequencies, which are not considered in the study.

Now, the transformation (19) reduces the Hamiltonian as:

$$
\begin{aligned}
& H_{2}^{\prime}=\frac{1}{2}\left(p_{1}^{\prime 2}+\omega_{1}^{2} q_{1}^{\prime 2}\right) \\
& -\frac{1}{2}\left(p_{2}^{\prime 2}+\omega_{2}^{2} q_{2}^{\prime 2}\right) \\
& +\frac{e \cos v}{1+e \cos v} \sum_{v+\mu=2} a_{v \mu}^{\prime} q_{1}^{\prime \prime 1} q_{2}^{\prime v 2} p_{1}^{\prime \mu 1} p_{2}^{\prime \mu 2}
\end{aligned}
$$


Where, $v=\left(v_{1}, v_{2}\right), \mu=\left(\mu_{1}, \mu_{2}\right)$ and such that

$v+\mu=v_{1}+v_{2}+\mu_{1}+\mu_{2}=2$.

The coefficients $a_{v \mu}^{\prime}$ are given as:

$$
\begin{aligned}
& a_{2000}^{\prime}=\left[(1-A) a_{1}^{2}+B a_{1}^{2} c_{1}+(1+C) a_{1}^{2} c_{1}^{2}\right] \\
& a_{0200}^{\prime}=\left[(1-A) a_{2}^{2}+B a_{2}^{2} c_{2}+(1+C) a_{2}^{2} c_{2}^{2}\right] \\
& a_{0020}^{\prime}=\left[(1+C) a_{1}^{2} b_{1}^{2}\right] \\
& a_{0002}^{\prime}=\left[(1+C) a_{2}^{2} b_{2}^{2}\right] \\
& a_{1100}^{\prime}=\left[\begin{array}{l}
2(1-A) a_{1} a_{2}+B a_{1} a_{2} c_{1} \\
+B a_{1} a_{2} c_{2}+2(1+C) a_{1} a_{2} c_{1} c_{2}
\end{array}\right] \\
& a_{1010}^{\prime}=\left[B a_{1}^{2} b_{1}+2(1+C) a_{1}^{2} b_{1} c_{1}\right] \\
& a_{1001}^{\prime}=\left[-B a_{1} a_{2} b_{2}-2(1+C) a_{1} a_{2} b_{2} c_{1}\right] \\
& a_{0110}^{\prime}=\left[B a_{1} a_{2} b_{1}+2(1+C) a_{1} a_{2} b_{1} c_{2}\right] \\
& a_{0011}^{\prime}=\left[-2(1+C) a_{1} a_{2} b_{1} b_{2}\right] \\
& a_{0101}^{\prime}=\left[-B a_{2}{ }^{2} b_{2}-2(1+C) a_{2}{ }^{2} b_{2} c_{2}\right]
\end{aligned}
$$

Now, using the transformation:

$$
\begin{aligned}
& q_{i}^{\prime}=\frac{1}{\sqrt{\omega_{i}}} \tilde{q}_{i} \\
& p_{i}^{\prime}=\sqrt{\omega_{i}} \tilde{p}_{i}
\end{aligned}
$$

The Hamiltonian gets transformed as:

$$
\tilde{H}_{2}^{(0)}=\frac{1}{2} \omega_{1}\left(\tilde{p}_{1}^{2}+\tilde{q}_{1}^{2}\right)-\frac{1}{2} \omega_{2}\left(\tilde{p}_{2}^{2}+\tilde{q}_{2}^{2}\right)
$$

and,

$$
\tilde{H}_{2}^{(1)}=\frac{e \cos v}{1+e \cos v} \sum_{v+\mu=2} \tilde{a}_{v \mu} \tilde{q}_{1}^{v 1} \tilde{q}_{2}^{v 2} \tilde{p}_{1}^{\mu 1} \tilde{p}_{2}^{\mu 2}
$$

where, $\tilde{a}_{v \mu}$ is given as :

$\tilde{a}_{2000}=\omega_{1} a_{2000}^{\prime} ; \tilde{a}_{0200}=\omega_{2} a_{0200}^{\prime} ; \tilde{a}_{0020}=\frac{1}{\omega_{1}} a_{0020}^{\prime} ;$

$\tilde{a}_{0002}=\frac{1}{\omega_{2}} a_{0002}^{\prime} ; \quad \tilde{a}_{1100}=\sqrt{\omega_{1} \omega_{2}} a_{1100}^{\prime} ; \tilde{a}_{1010}=a_{1010}^{\prime} ;$

$\tilde{a}_{1001}=\sqrt{\frac{\omega_{1}}{\omega_{2}}} a_{1001}^{\prime} ; \tilde{a}_{0110}=\sqrt{\frac{\omega_{2}}{\omega_{1}}} a_{0110}^{\prime} ; \tilde{a}_{0011}=\frac{1}{\sqrt{\omega_{1} \omega_{2}}} a_{0011}^{\prime} ;$

$\tilde{a}_{0101}=a_{0101}^{\prime}$

Now, introducing the complex conjugate variables given by:

$$
q_{j}^{\prime \prime}=\tilde{p}_{j}+i \tilde{q}_{j} ; p_{j}^{\prime \prime}=\tilde{p}_{j}-i \tilde{q}_{j} ;(j=1,2)
$$

$$
\begin{aligned}
& H_{2}^{\prime \prime}=i \omega_{1} q_{1}^{\prime \prime} p_{1}^{\prime \prime}+i \omega_{2} q_{2}^{\prime \prime} p_{2}^{\prime \prime} \\
& +2 i \frac{e \cos v}{1+e \cos v} \sum_{v+\mu=2} a_{v \mu}^{\prime \prime} q_{1}^{\prime \prime} v q_{2}^{\prime \prime} v 2 p_{1}^{\prime \prime} \mu 1 p_{2}^{\prime \prime} \mu 2
\end{aligned}
$$

The coefficients in $H_{2}^{\prime \prime}$ are such that $a_{v \mu}^{\prime \prime}=\bar{a}_{v \mu}^{\prime \prime}$

$$
\begin{aligned}
& a_{2000}^{\prime \prime}=\frac{1}{4}\left\{-\tilde{a}_{2000}+\tilde{a}_{0020}-i \tilde{a}_{1010}\right\} \\
& a_{0200}^{\prime \prime}=\frac{1}{4}\left\{-\tilde{a}_{0200}+\tilde{a}_{0002}-i \tilde{a}_{0101}\right\} \\
& a_{1100}^{\prime \prime}=\frac{1}{4}\left\{-\tilde{a}_{1100}+\tilde{a}_{0011}-i \tilde{a}_{1001}-i \tilde{a}_{0110}\right\} \\
& a_{1001}^{\prime \prime}=\frac{1}{4}\left\{-\tilde{a}_{1100}+\tilde{a}_{0011}-i \tilde{a}_{1001}-i \tilde{a}_{0110}\right\} \\
& a_{1010}^{\prime \prime}=\frac{1}{2}\left\{\tilde{a}_{2000}+\tilde{a}_{0020}\right\} \\
& a_{0101}^{\prime \prime}=\frac{1}{2}\left\{\tilde{a}_{0200}+\tilde{a}_{0002}\right\}
\end{aligned}
$$

Next, to reduce the Hamiltonian (30) to normal form in complex conjugate variables given as:

$$
H_{2}^{*}\left(q_{j}^{* *}, p_{j}^{* *}\right)=i \lambda_{1} q_{1}^{* *} p_{1}^{* *}+i \lambda_{2} q_{2}^{* *} p_{2}^{* *}
$$

Finding a transformation of the type:

$$
\left(q_{j}^{\prime \prime}, p_{j}^{\prime \prime}\right) \rightarrow\left(q_{j}^{* *}, p_{j}^{* *}\right)
$$

defined by the generating function:

$$
q_{1}^{\prime \prime} p_{1}^{* *}+p_{2}^{\prime \prime} q_{2}^{* *}+S\left(q_{1}^{\prime \prime}, q_{2}^{\prime \prime}, p_{1}^{* *}, p_{2}^{* *}, v\right)
$$

where

$$
S=\sum_{v+\mu=2} s_{v \mu} q_{1}^{\prime \prime v 1} q_{2}^{\prime \prime v 2} p_{1}^{* *} \mu 1 p_{2}^{* *} \mu 2
$$

$s_{v \mu}$ are $2 \pi$ periodic functions of $v$. The relation between the variables are given as:

$$
\begin{aligned}
& q_{j}^{* *}=q_{j}^{\prime \prime}+\frac{\partial s}{\partial p_{j}^{* *}} \\
& p_{j}^{\prime \prime}=p_{j}^{* *}+\frac{\partial s}{\partial q_{j}^{\prime \prime}}
\end{aligned}
$$

Using the above relation, we have the equation:

$$
H_{2}^{* *}\left(q_{j}^{\prime \prime}+\frac{\partial s}{\partial p_{j}^{* *}}, p_{j}^{* *}, v\right)-H_{2}^{\prime \prime}\left(q_{j}^{\prime \prime}, p_{j}^{* *}+\frac{\partial s}{\partial q_{j}^{\prime \prime}}, v\right)=\frac{\partial s}{\partial v}
$$

Now, expanding the Hamiltonian using Taylor's theorem, and equating the equal powers on both sides:

which reduces the Hamiltonian, $H_{2}^{\prime \prime}=2 i \tilde{H}_{2}$, where, 


$$
\begin{aligned}
& i \lambda_{1} q_{1}^{\prime \prime} p_{1}^{* *}+i \lambda_{2} q_{2}^{\prime \prime} p_{2}^{* *} \\
& +i \sum_{v+\mu=2}\left(\mu_{1} \lambda_{1}+\mu_{2} \lambda_{2}\right) s_{v \mu} q_{1}^{\prime \nu 1} q_{2}^{\prime \prime v 2} p_{1}^{* *} \mu 1 p_{2}^{* *} \mu 2 \\
& -i \omega_{1} q_{1}^{\prime \prime} p_{1}^{* *}-i \omega_{2} q_{2}^{\prime \prime} p_{2}^{* *} \\
& -2 i \frac{e \cos v}{1+e \cos v} \sum_{v+\mu=2} a_{v \mu}^{\prime \prime} q_{1}^{\prime \prime} v 1 q_{2}^{\prime \prime} v 2 p_{1}^{* *} \mu 1_{p_{2}^{* *}}^{* * 2} \\
& -i \sum_{v+\mu=2}\left(v_{1} \omega_{1}-v_{2} \omega_{2}\right) s_{v \mu} q_{1}^{\prime \prime \nu 1} q_{2}^{\prime \prime} v 2 p_{1}^{* *} \mu 1 p_{2}^{* *} \mu 2 \\
& =\sum_{v+\mu=2} \frac{d s}{d v} q_{1}^{\prime \prime}{ }^{\prime \prime} q_{2}^{\prime \prime} v 2 p_{1}^{* *} \mu 1 p_{2}^{* *} \mu 2
\end{aligned}
$$

Taking the terms up to second order in $e$ :

$$
\begin{aligned}
& i \lambda_{1} q_{1}^{\prime \prime} p_{1}^{* *}+i \lambda_{2} q_{2}^{\prime \prime} p_{2}^{* *} \\
& +i \sum\left(\mu_{1} \lambda_{1}+\mu_{2} \lambda_{2}\right)\left(e s{ }^{(1)}+e_{s}^{2(2)}\right) \\
& -i \omega_{1} q_{1}^{\prime \prime} p_{1}^{* *}-i \omega_{2} q_{2}^{\prime \prime} p_{2}^{* *} \\
& -2 i\left[e \cos v-\frac{e^{2}}{2}(1+\cos 2 v)\right] \sum_{v+\mu=2} a_{v \mu}^{\prime \prime} q_{1}^{\prime \prime} v 1_{2}^{\prime \prime} 2^{* *} p_{1}^{*} \mu 1 p_{2}^{* *} \mu 2 \\
& -i \sum_{v+\mu=2}\left(v_{1} \omega_{1}-v_{2} \omega_{2}\right)(e s \\
& =e \frac{d s}{d v}+e^{2} \frac{d s}{d v}(2)
\end{aligned}
$$

where

$$
s_{v \mu}=e \sum s^{(1)}+e^{2} \sum s^{(2)}
$$

and taking:

$$
\lambda_{i}=\lambda_{i}^{(0)}+e \lambda_{i}^{(1)}+e^{2} \lambda_{i}^{(2)}+
$$

Now, equating the equal powers of $e$ on both sides of equation (38), we have:

$$
\begin{aligned}
& \lambda_{1}^{(0)}=\omega_{1} ; \lambda_{2}^{(0)}=\omega_{2} ; i \lambda_{1}^{(1)}+i \lambda_{1}^{(2)} \\
& +i \sum\left(\mu_{1} \lambda_{1}^{(0)}+\mu_{2} \lambda_{2}^{(0)}\right) s^{(1)}-2 i a_{v \mu}^{\prime \prime} \cos v \\
& -i \sum\left(v_{1} \mu_{1}-v_{2} \mu_{2}\right)=\frac{d s^{(1)}}{d v}
\end{aligned}
$$

From which we get the relation:

$$
\frac{d s}{d v}+i\left[\left(v_{1}-\mu_{1}\right) \omega_{1}-\left(v_{2}-\mu_{2}\right) \omega_{2}\right]=-2 i a_{v \mu}^{\prime \prime} \cos v
$$

Solving, the above equation, we have:

$$
s_{v \mu}^{(1)}=\frac{2 i a_{v \mu}^{\prime \prime}\left[\sin v+i\left\{\left(v_{1}-\mu_{1}\right) \omega_{1}-\left(v_{2}-\mu_{2}\right) \omega_{2}\right\} \cos v\right]}{\left[\left(v_{1}-\mu_{1}\right) \omega_{1}-\left(v_{2}-\mu_{2}\right) \omega_{2}\right]^{2}-1}
$$

By virtue of periodicity of $s_{1010}^{(1)}$ and $s_{0101}^{(1)}$, we have $\lambda_{1}^{(1)}=\lambda_{2}^{(1)}=0$. So, equation (43) completely determines the complex-valued generating function $\mathrm{S}$ correct upto $\mathrm{O}[e]$.
Finally, reducing the Hamiltonian $\tilde{H}_{2}=\tilde{H}_{2}^{(0)}+\tilde{H}_{2}^{(1)}$ to the normal form given by:

$$
H_{2}=\frac{1}{2} \lambda_{1}\left(q^{* 2}+p_{1}^{* 2}\right)+\frac{1}{2} \lambda_{2}\left(\begin{array}{c}
*^{2} 2 \\
2
\end{array}\right.
$$

With the real-valued transformation $\left(\tilde{q}_{j}, \tilde{p}_{j}\right) \rightarrow\left(q_{j}^{*}, p_{j}^{*}\right)$ by means of generating function $\tilde{q}_{1} p_{1}^{*}+\tilde{q}_{2} p_{2}^{*}+K\left(\tilde{q}_{j}, p_{j}^{*}, v\right)$, up to $\mathrm{O}[e]$, using the transformation formula given by:

$q_{j}^{*}=\tilde{q}_{j}+\frac{\partial K}{\partial p_{j}^{*}}, \tilde{p}_{j}=p_{j}^{*}+\frac{\partial K}{\partial q_{j}^{*}}$

which can be written using Implicit function theorem as:

$$
\tilde{q}_{j}=q_{j}^{*}-\frac{\partial K}{\partial p_{j}^{*}}, \tilde{p}_{j}=p_{j}^{*}+\frac{\partial K}{\partial q_{j}^{*}}
$$

Now, using equation (33), up to first order terms in $e$, we have the relation:

$$
q_{j}^{\prime \prime}=q_{j}^{* *}-\frac{\partial S^{* * *}}{\partial p_{j}^{* * *}}, p_{j}^{\prime \prime}=p_{j}^{* *}+\frac{\partial S^{* *}}{\partial q_{j}^{* *}}
$$

where, $S^{* *}=S^{(1)}\left(q^{* *}, p^{* *}, v\right)$. Now, using the relations:

$q_{j}^{\prime \prime}=\tilde{p}_{j}+i \tilde{q}_{j}, p_{j}^{\prime \prime}=\tilde{p}_{j}-i \tilde{q}_{j}$

$q_{j}^{* *}=p_{j}^{*}+i q_{j}^{*}, p_{j}^{* *}=p_{j}^{*}-i q_{j}^{*}$

Let $S^{(1)}=W$, so from equation(46) we have:

$\tilde{q}_{j}=q_{j}^{*}-\frac{1}{2 i} \frac{\partial W}{\partial p_{j}^{*}}, \tilde{p}_{j}=p_{j}^{*}+\frac{1}{2 i} \frac{\partial W}{\partial q_{j}^{*}}$

From, equations (46) and (48), we have:

$K=\frac{1}{2 i} W$

where, the function $K=\sum k_{\nu \mu} q_{1}^{*}{ }^{*}{ } q_{2}^{*}{ }^{*}{ }_{p_{1}}^{*} \mu 1{ }_{p_{2}}^{* \mu 2}$ is a real valued function. The coefficients are given by using the relations (25) and (43) as:

$k_{2000}=\frac{1}{2 i}\left(-s_{2000}^{(1)}-s_{0020}^{(1)}+s_{1010}^{(1)}\right)$

$k_{0200}=\frac{1}{2 i}\left(-s_{0200}^{(1)}-s_{0002}^{(1)}+s_{0101}^{(1)}\right)$;

$k_{0020}=\frac{1}{2 i}\left(s_{2000}^{(1)}-s_{0020}^{(1)}+s_{1010}^{(1)}\right)$;

$k_{0002}=\frac{1}{2 i}\left(s_{0200}^{(1)}-s_{0002}^{(1)}+s_{0101}^{(1)}\right)$; 


$$
\begin{aligned}
& k_{1100}=\frac{1}{2 i}\left(s_{1100}^{(1)}+s_{1001}^{(1)}+s_{0110}^{(1)}-s_{0011}^{(1)}\right) \\
& k_{1010}=\left(s_{2000}^{(1)}-s_{0020}^{(1)}\right) ; \\
& k_{1001}=\frac{1}{2}\left(s_{1100}^{(1)}+s_{1001}^{(1)}-s_{0110}^{(1)}-s_{0011}^{(1)}\right) \\
& k_{1001}=\frac{1}{2}\left(s_{1100}^{(1)}+s_{1001}^{(1)}-s_{0110}^{(1)}-s_{0011}^{(1)}\right) \\
& k_{0101}=\left(s_{0200}^{(1)}-s_{0002}^{(1)}\right) ; \\
& k_{0110}=\frac{1}{2}\left(s_{1100}^{(1)}-s_{1001}^{(1)}+s_{0110}^{(1)}-s_{0011}^{(1)}\right) \\
& k_{0011}=\frac{1}{2 i}\left(s_{1100}^{(1)}+s_{1001}^{(1)}+s_{0110}^{(1)}+s_{0011}^{(1)}\right)
\end{aligned}
$$

Thus, the Hamiltonian $\mathrm{H}_{2}$ has been transformed to normal form given by equation (44), correct up to first order in eccentricity $e$. This is obtained using equations (19), (25) and (46) and the corresponding coefficients of the generating function $\mathrm{K}$ are given by (50).

\section{Resonance cases}

For the study of stability, we have to examine the presence of resonances. For this, taking third order terms and applying the KAM theorem. So, considering the value of $\lambda_{1}$ and $\lambda_{2}$ up to second order of $e$. As the value $\mathrm{f} \lambda_{1}^{(1)}=\lambda_{2}^{(1)}=0$, so the quantities $\lambda_{1}^{(2)}$ and $\lambda_{2}^{(2)}$ are found by the periodicity conditions of the functions $s_{1010}^{(2)}$ and $s_{0101}^{(2)}$. Using (38) and equating the terms of $e^{2}$, we have:

$$
\frac{d s_{1010}^{(2)}}{d v}=-2 i \cos v\left(\begin{array}{c}
4 a_{0020}^{\prime \prime}{ }_{2000}^{(1)} \\
+a_{1010^{\prime \prime}}^{s_{1010}} \\
+a_{1001}^{\prime \prime}{ }_{1001}^{(1)} \\
+a_{0110}^{\prime \prime}{ }_{1100}^{(1)}
\end{array}\right)
$$

$+2 i \cos ^{2} v a_{1010}^{\prime \prime}+i \lambda_{1}^{(2)}$

$\frac{d s_{0101}^{(2)}}{d v}=-2 i \cos v\left(\begin{array}{c}4 a_{0002}^{\prime \prime} s_{0200}^{(1)} \\ +a_{0110^{\prime \prime}}^{s_{1001}^{(1)}} \\ +a_{0101}^{\prime \prime} \\ (1) \\ +a_{0011}^{\prime \prime}\end{array}\right.$

$+2 i \cos ^{2} v a_{0101}^{\prime \prime}+i \lambda_{2}^{(2)}$

Now, using equation (43) along with the condition of periodicity of $s_{1010}^{(2)}$ and $s_{0101}^{(2)}$, the values of $\lambda_{1}^{(2)}$ and $\lambda_{2}^{(2)}$ using equations (14) and (21) are given as:

$$
\begin{aligned}
& \lambda_{1}^{(2)}=-\frac{\omega_{1} \omega_{2}^{2}\left(6 \omega_{1}^{2}-7\right)}{4\left(4 \omega_{1}^{2}-1\right)\left(2 \omega_{1}^{2}-1\right)} ; \\
& \lambda_{2}^{(2)}=\frac{\omega_{1}^{2} \omega_{2}\left(6 \omega_{2}^{2}-7\right)}{4\left(4 \omega_{2}^{2}-1\right)\left(2 \omega_{2}^{2}-1\right)}
\end{aligned}
$$

Now, let the value of $\mu$ giving the resonance be:

$$
k_{1} \lambda_{1}+k_{2} \lambda_{2}=N
$$

For small values of $e$. The value of $\mu$ correct up to order of $e^{2}$ is given as:

$$
\mu=\mu^{(0)}+e^{2} \mu^{(2)}
$$

where $\mu^{(0)}$ is the value of $\mu$ for $e=0$ and $\mu^{(2)}$ is the value of $\mu$ for $e \neq 0$ taken up to order of $e^{2}$, given by the equation, following Usha et. al[27] is :

$$
\mu(e)=\left[\begin{array}{l}
27+\frac{4311}{16} \sigma_{1} \\
-\frac{5469}{16} \sigma_{2} \\
+\left(1-e^{2}\right)^{\frac{1}{2}}\left(16 \delta+45 \sigma_{1}-69 \sigma_{2}\right)
\end{array}\right\} \pm
$$

$\left\{\begin{array}{l}-1971+300 \delta+\frac{228457}{8} \sigma_{1}-\frac{642583}{8} \sigma_{2} \\ +e^{2}\left(1728-192 \delta-27800 \sigma_{1}+37304 \sigma_{2}\right) \\ +\left(1-e^{2}\right)^{\frac{1}{2}}\left(2592-1632 \delta-4377 \sigma_{1}+59330 \sigma_{2}\right)\end{array}\right\}$

$\div 2\left[\begin{array}{l}27-3 \delta-\frac{3379}{8} \sigma_{1}+\frac{4567}{8} \sigma_{2} \\ +8\left(1-e^{2}\right)\left(\sigma_{1}-\sigma_{2}\right)\end{array}\right]$

Taking, $\lambda_{i}=\lambda_{i}\left(\mu^{(0)}+e^{2} \mu^{(2)}\right), \mathrm{i}=1,2$ and expanding by Taylor's theorem,

$$
\lambda_{i}=\lambda_{i}^{(0)}+e^{2} \lambda_{i}^{(2)}+e^{2} \mu^{(2)}\left(\frac{d \lambda_{i}}{d \mu}\right)_{0}, \mathrm{i}=1,2
$$

where, $\lambda_{1}{ }^{(2)}, \lambda_{2}{ }^{(2)}$ are given by equation by (52). Now, substituting the above obtained values in equation (53) and equating the coefficient of $e^{2}$ to zero, we have:

$$
\mu^{(2)}=\frac{k_{1} \lambda_{1}^{(2)}+k_{2} \lambda_{2}^{(2)}}{k_{2} \frac{d \omega_{2}}{d \mu}-k_{1} \frac{d \omega_{1}}{d \mu}}
$$

The value of $\mu^{(2)}$ is calculated on putting the value $\mu=\mu^{(0)}$ calculated from equation (54) and substituting different values of $k_{1}, k_{2}, N$ in the above equation, the values of third order resonances are calculated. 
Let us consider, the third order resonance occur for:

$3 \lambda_{2}=-1 ; 2 \lambda_{1}+\lambda_{2}=0 ; 2 \lambda_{1}+\lambda_{2}=1 ; \lambda_{1}-2 \lambda_{2}=2$.

Calculating the values of $\mu^{(0)}$ and $\mu^{(2)}$ at $e=0$ for different resonance cases and plotting the graphs we can analyze the stability of the system. The graphs have been plotted using MATLAB and the calculations have been verified using the software Mathematica.

\section{Conclusion}

The resonance cases and the linear stability of the elliptic restricted three body problem with radiating primary and triaxial secondary has been analyzed. It is observed from the graphs that the linear stability is satisfied for the resonance cases $3 \lambda_{2}=-1$ and $2 \lambda_{1}+\lambda_{2}=0$ (Figures1 \& 2). On the other hand, the condition of linear stability does not hold for the resonance cases $\lambda_{1}-2 \lambda_{2}=2$ and $2 \lambda_{1}+\lambda_{2}=1$ which are clear from the graphs (Figures $3 \& 4$ ). These results are in confirmation with Kumar [20].

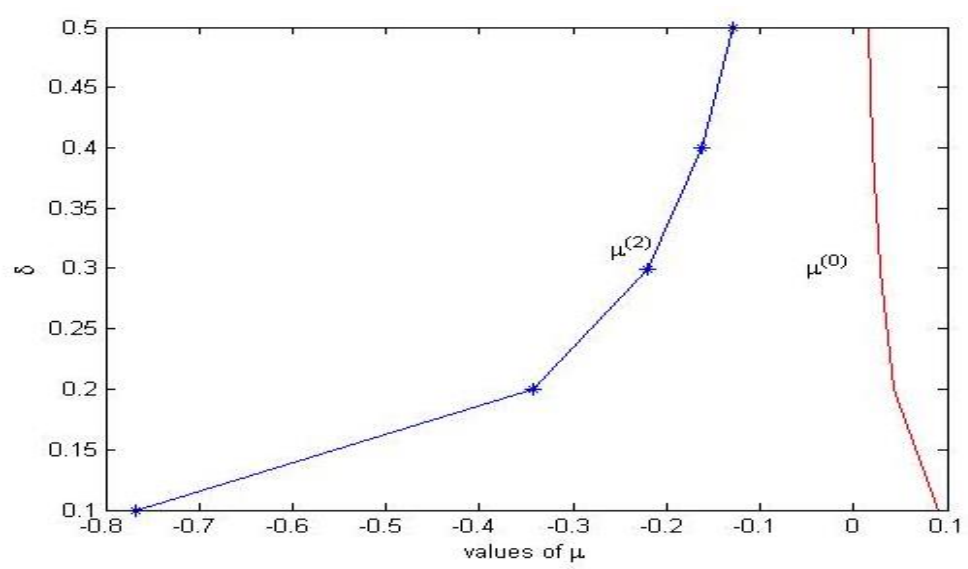

Fig. 1: Analysis of Stability For $3 \lambda_{2}=-1$

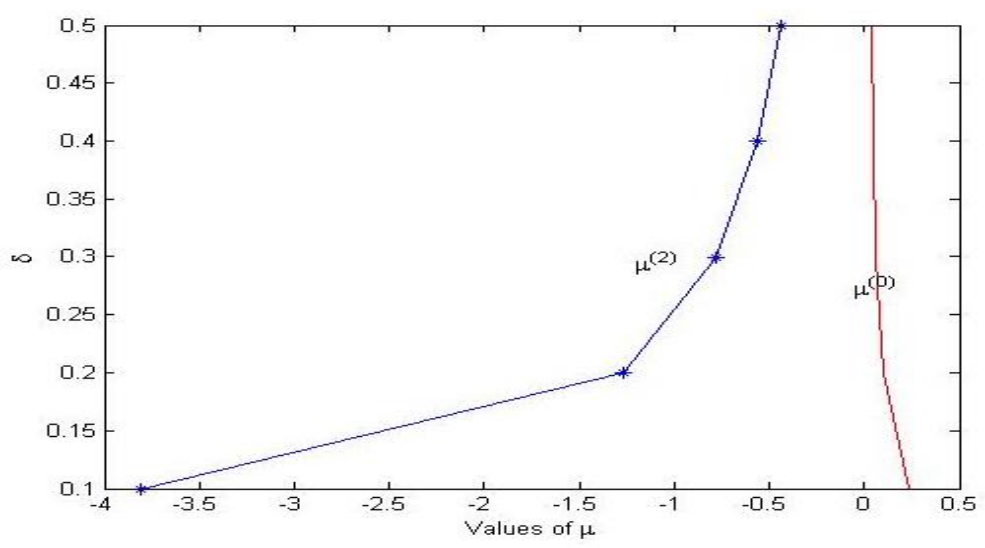

Fig. 2: Analysis of Stability for $2 \lambda_{1}+\lambda_{2}=0$.

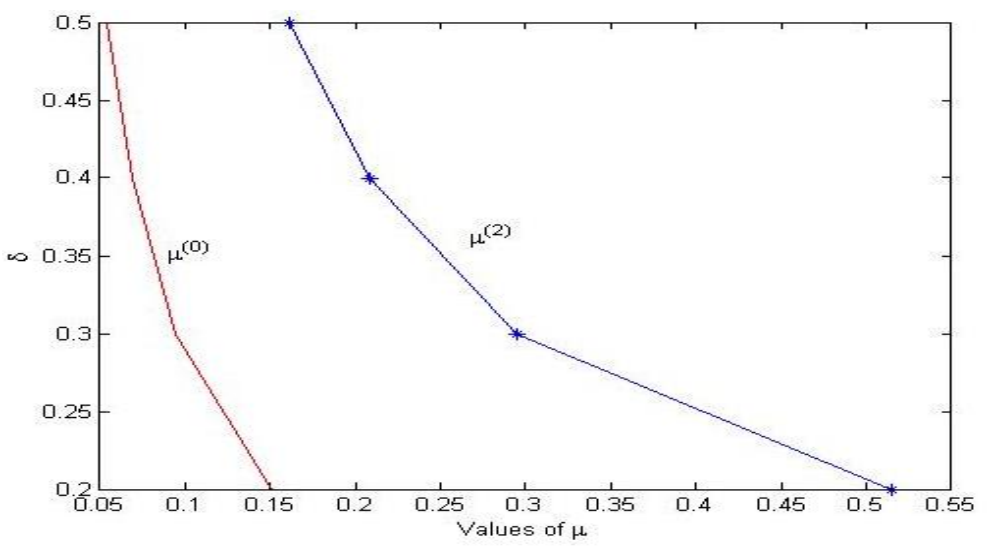

Fig. 3: Analysis of Stability for $2 \lambda_{1}+\lambda_{2}=1$. 


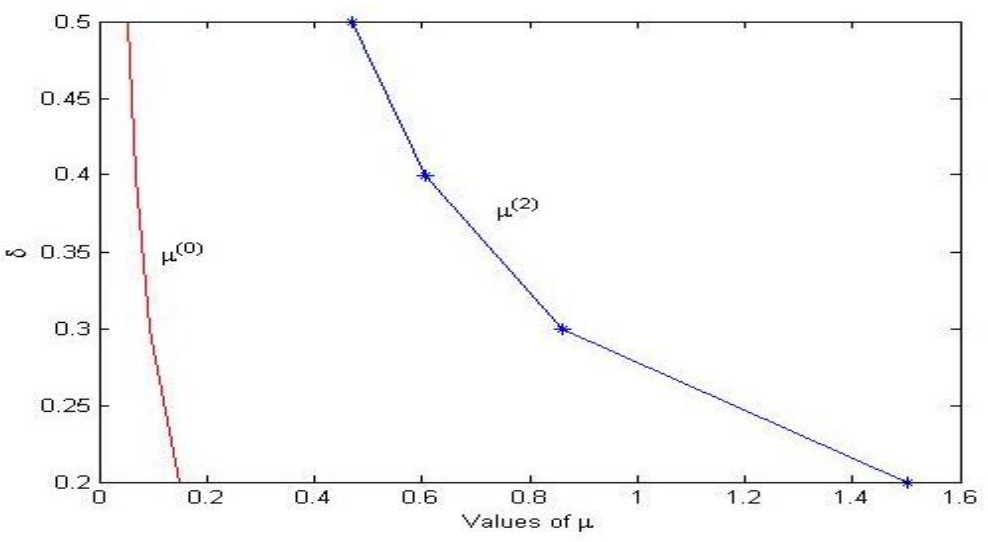

Fig. 4: Analysis of Stability for $\lambda_{1}-2 \lambda_{2}=2$.

\section{References}

[1] Arnold, V. I.; Stability of equilibrium position of a Hamiltonian system of ordinary differential equations I general elliptic case; Doklady Akademii Nauk SSR; 137(2), 255, 1961

[2] Ammar, M. K.; The effect of solar radiation pressure on the Lagrangian points in the elliptic restricted three-body problem; Astrophysics and Space Science; 313(4):393-408, 2008. http://dx.doi.org/10.1007/s10509-007-9709-z.

[3] Baoyin, Hexi. McInnes, Colin, R.; Solar sail equilibria in the elliptical restricted three-body problem. Journal of Guidance, Control, and Dynamics, 29(3):538-543, 2006 http://dx.doi.org/10.2514/1.15596.

[4] Bennet, A.; Characteristic exponents of the five equilibrium solutions in the elliptically restricted problem; ICARUS; 4(2), 177-187, 1965. http://dx.doi.org/10.1016/0019-1035(65)90060-6

[5] C Beaug'e., S Ferraz-Mello., Michtchenko, T., A.,; Extrasolar planets in mean-motion resonance: apses alignment and asymmetric stationary solutions. The Astrophysical Journal, 593(2):1124, 2003. http://dx.doi.org/10.1086/376568.

[6] Broucke, R.; Stability of periodic orbits in the elliptic, restricted three-body problem; AIAA journal; 7(6):1003-1009, 1969. http://dx.doi.org/10.2514/3.5267.

[7] Biggs, James, D., Colin, R., McInnes., Thomas, Waters., ; Control of solar sail periodic orbits in the elliptic three-body problem. Journal of Guidance, Control, and Dynamics, 32(1):318-320, 2009a. http://dx.doi.org/10.2514/1.38362.

[8] Biggs, James, D., Colin, R., McInnes., Thomas, Waters., ; Solar sail formation flying for deep-space remote sensing; Journal of Guidance, Control, and Dynamics,; 46(3):670-678, 2009b.

[9] Chandra, Navin., Kumar, Ranjeet.,; Effect of oblateness on the nonlinear stability of the triangular liberation points of the restricted three-bo dy problem in the presence of resonances. Astrophysics and Space $\quad$ Science, 291(1):1-19, 2004. http://dx.doi.org/10.1023/B:ASTR.0000029925.13391.7d.

[10] Choudhry, Manju., Choudhry, R , K. , ; On the stability of triangular libration points taking into account the light pressure for the circular restricted problem of three bodies. Celestial mechanics, 36(2):165-190, 1985. http://dx.doi.org/10.1007/BF01230650.

[11] Danby, J, M, A.; Stability of the triangular points in the elliptic restricted problem of three bodies; the Astronomical Journal, 69:165, 1964.

[12] S Ferraz-Mello. Averaging the elliptic asteroidal problem near a first-order resonance. The Astronomical Journal, 94:208-212, 1987. http://dx.doi.org/10.1086/114465.

[13] Hadjidemetriou, John, D., The elliptic restricted problem at the 3: 1 resonance. Celestial Mechanics and Dynamical Astronomy; 53(2):151-183, 1992. http://dx.doi.org/10.1007/BF00049463.

[14] Hadjidemetriou, John, D., Resonant motion in the restricted three body problem. In Qualitative and Quantitative Behaviour of Planetary Systems, pages 201-219. Springer, 1993. http://dx.doi.org/10.1007/978-94-011-2030-2 19.

[15] Henrard, Jacques, Caranicolas, N, D., A perturbative treatment of the 2:1 Jovian resonance; ICARUS; 69(2):266-279, 1987. http://dx.doi.org/10.1016/0019-1035(87)90105-9.

[16] Henrard, Jacques, Caranicolas, N, D., Motion near the 3/1 resonance of the planar elliptic restricted three body problem; Celestial Mechanics and Dynamical Astronomy; 47(2):99-121, 1989. http://dx.doi.org/10.1007/BF00051201.
[17] Henrard, Jacques; A semi-numerical perturbation method for separable Hamiltonian systems; Celestial Mechanics and Dynamical Astronomy 49(1):43-67,

1990. http://dx.doi.org/10.1007/BF00048581.

[18] Kamel, A, A., Nayfeh, A, H.,; Stability of the triangular points in the elliptic restricted problem of three bo dies. AIAA Journal, 8(2):221-223, 1970. http://dx.doi.org/10.2514/3.5646.

[19] Katsiaris, G.; The three-dimensional elliptic problem; In Recent Advances in Dynamical Astronomy, pages 118-134; Springer; 1973. http://dx.doi.org/10.1007/978-94-010-2611-6_12.

[20] Kumar, V., Choudhry, R, K.,; Linear stability and the resonance cases for the triangular libration points for the doubly photogravitational elliptic restricted problem of three bodies; Celestial Mechanics and Dynamical Astronomy, 46(1):59-77, 1989. http://dx.doi.org/10.1007/BF02426713.

[21] McCuskey, Sidney, Wilcox. ; Introduction to Celestial Mechanics; Reading; Mass.; Addison-Wesley Pub. Co. [1963], 1, 1963.

[22] Narayan, A., Shrivastava, Amit. , Existence of resonance stability of triangular equilibrium points in circular case of the planar elliptical restricted three-body problem under the oblate and radiating primaries around the binary system. Advances in Astronomy, 2014, 2014a.

[23] Narayan, A., Usha, T.; Stability of triangular equilibrium points in the elliptic restricted problem of three bodies with radiating and triaxial primaries. Astrophysics and Space Science, 351(1):135-142, 2014b. http://dx.doi.org/10.1007/s10509-014-1818-x.

[24] Subba Rao, P, V., Sharma, Ram Krishan.; Effect of oblateness on the non-linear stability of L4 in the restricted three-body problem. Celestial Mechanics and Dynamical Astronomy, 65(3):291-312,

[25] Szebehely, V., Theory of orbits: The restricted three body problem, 1967.

[26] Thakur, A, P. Singh, R, B.; Stability of the triangular libration points of the circular restricted problem in the presence of resonances. Celestial Mechanics and Dynamical Astronomy, 66(2):191-202, 1996. http://dx.doi.org/10.1007/BF00054964.

[27] Usha, T., Narayan, A., Ishwar, B.; Effects of radiation and triaxiality of primaries on triangular equilibrium points in elliptic restricted three body problem; Astrophysics and Space Science; 349(1):151164, 2014 http://dx.doi.org/10.1007/s10509-013-1655-3. 